\title{
Los procesos de nasalización en bribri en el marco de la teoría de la optimidad
}

\author{
Haakon S. Krohn
}

Krohn, H. S. (2021). Los procesos de nasalización en bribri en el marco de la teoría de la optimidad. Revista de Filología y Lingüística de la Universidad de Costa Rica, 47(2), e46626. doi: https://doi.org/10.15517/rfl.v47i2.46626

\section{(9) $\mathbb{P Q \Theta}$}

Doi: https://doi.org/10.15517/rfl.v47i2.46626

URL: https://revistas.ucr.ac.cr/index.php/filyling/index 
Revista de Filología y Lingüística de la Universidad de Costa Rica

ISSN: 0377-628X

ISSN: 2215-2628

filyling@gmail.com

Universidad de Costa Rica

Costa Rica

\section{Los procesos de nasalización en bribri en el marco de la teoría de la optimidad ${ }^{1}$}

Krohn, Haakon S.

Los procesos de nasalización en bribri en el marco de la teoría de la optimidad ${ }^{1}$

Revista de Filología y Lingüística de la Universidad de Costa Rica, vol. 47, núm. 2, e46626, 2021

Universidad de Costa Rica, Costa Rica

Disponible en: http://www.redalyc.org/articulo.oa?id=33266553001

DOI: https://doi.org/10.15517/rfl.v47i2.46626

\section{(c) $(1) \Theta($}

Esta obra está bajo una Licencia Creative Commons Atribución-NoComercial-SinDerivar 3.0 Internacional. 


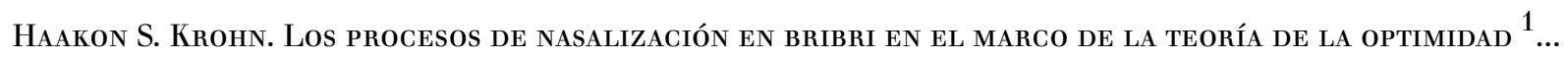

Lingüística

\title{
Los procesos de nasalización en bribri en el marco de la teoría de la optimidad ${ }^{1}$
}

The Nasalization Processes in Bribri in the Framework of the Optimality Theory

Haakon S. Krohn

Universidad de Costa Rica, San José, Costa Rica

hkrohn@gmail.com

iD https://orcid.org/0000-0002-8888-8512

\author{
DOI: https://doi.org/10.15517/rfl.v47i2.46626
//www.redalyc.org/articulo.oa?id=33266553001 \\ Redalyc: http://www.redalyc.org/articulo.oa?id=33266553001
}

Recepción: 10 Diciembre 2020

Aprobación: 16 Febrero 2021

\section{Resumen:}

A nivel fonológico, la lengua bribri no posee consonantes nasales, pero sí cuenta con una serie de fonemas vocálicos nasales que contrastan con las vocales orales. Tres procesos fonológicos de nasalización diferentes afectan tanto consonantes sonoras como vocales átonas y producen alófonos nasales: armonía nasal con propagación regresiva, nasalización progresiva de codas silábicas y nasalización en codas silábicas como resultado de hipersonorización. Estos tres procesos se analizan en el presente artículo dentro del marco de la teoría de la optimidad. Se plantea que dos restricciones de alineamiento distintas son los propulsores de la armonía nasal y la nasalización progresiva, y que la hipersonorización, dependiendo del caso, es consecuencia de una restricción que penaliza el fono [b] en coda silábica o de una que penaliza consonantes sonoras geminadas.

Palabras Clave: lengua bribri, fonología, teoría de la optimidad, armonía nasal, hipersonorización.

\section{Abstract:}

At the phonological level, the Bribri language does not possess nasal consonants, but it does have a series of nasal vowel phonemes that contrast with the oral vowels. Three phonological nasalization processes affect voiced consonants and unstressed vowels and produce nasal allophones: nasal harmony with regressive spreading, progressive nasalization of syllable codas and nasalization in codas as a result of hypervoicing. These three processes are analyzed in the present paper within the framework of the Optimality Theory. I suggest that two different alignment constraints are the instigators of the nasal harmony and the progressive nasalization, and that the hypervoicing, depending on the case, is a consequence of a constraint that penalizes $[\mathrm{b}]$ in codas or of one that penalizes geminated voiced consonants.

KEYWORDS: Bribri language, phonology, Optimality Theory, nasal harmony, hypervoicing.

\section{INTRODUCCIÓN}

El bribri, una lengua chibchense ${ }^{2}$ hablada en Costa Rica y Panamá, presenta una particularidad fonológica que es relativamente poco común en las lenguas del mundo: no posee fonemas consonánticos nasales. Sin embargo, sí ocurren consonantes nasales a nivel fonético, como resultado de tres procesos fonológicos diferentes: armonía nasal con propagación regresiva, nasalización progresiva a codas silábicas y nasalización por hipersonorización en codas silábicas. Además, el primer proceso también puede afectar vocales inacentuadas. En el presente artículo se realiza un análisis de estos fenómenos desde la perspectiva de la teoría de la optimidad, en lo que constituye la primera aplicación de dicha teoría a datos de una lengua chibchense.

En cuanto a la armonía nasal, consideramos que el impulsor es una restricción de alineamiento que favorece que el rasgo [+nasal] de una vocal fonológicamente nasal sea asociado con todos los segmentos entre dicha vocal y el extremo izquierdo de la palabra, y que esta propagación es bloqueada por consonantes sordas y vocales orales acentuadas, que son los únicos segmentos que no pueden nasalizarse en bribri. Por otro lado, la nasalización progresiva debe de ser incitada por otra restricción de alineamiento, cuyo dominio es el morfema. Por último, la nasalización por hipersonorización en coda silábica tiene lugar bajo condiciones más restrictivas, lo cual indica que tienen que existir restricciones muy específicas con referencia a consonantes 
en esta posición; además, una prohibición de consonantes sonoras geminadas es activa y produce el mismo fenómeno en palabras formadas por una raíz reduplicada.

\section{Marco teórico}

\subsection{La teoría de la optimidad}

El objetivo principal del presente trabajo es hacer uso de la teoría de la optimidad para desarrollar un análisis novedoso y contemporáneo de los procesos de nasalización en bribri. Se trata de una teoría del lenguaje humano muy renombrada que no solo es relevante para la fonología, sino para prácticamente cualquier área de la lingüística. Aun así, con anterioridad a la presente obra, no se ha publicado ningún análisis de una lengua chibchense dentro de este marco teórico tan influyente en la lingüística actual. Dada esta índole particular del estudio, se ofrece aquí una breve exposición de la teoría.

La teoría de la optimidad consiste en un modelo generativo no derivacional propuesto por Prince y Smolensky (1993/2004). Rompe con la tradición de reglas de reescritura iniciada por Chomsky y Halle (1968) y plantea, en cambio, un proceso de selección del educto (“output”) más óptimo de todos los posibles para un aducto ("input") dado. Esto quiere decir que el modelo no involucra ninguna transformación, sino que, en lo que concierne a los procesos fonológicos, la forma fonética (el educto) es la que más se adecua a la forma fonológica (el aducto) de acuerdo con la jerarquía de restricciones fonológicas de cada lengua.

El modelo consiste en dos componentes fundamentales: la función GEN ("Generator") genera todos los candidatos posibles, los cuales, en teoría, constituyen una cantidad infinita (McCarthy, 2008, p. 17), mientras que la función EVAL ("Evaluator") es responsable de evaluar los candidatos y escoger el óptimo. Además, se postula que existe un conjunto de restricciones ("constraints") universales, las cuales están jerarquizadas de manera diferente en cada lengua. Uno de los objetivos de las investigaciones que se adscriben a esta teoría es identificar dichas restricciones.

Ya que las restricciones son compartidas por todas las lenguas, la variación interlingüística tiene que derivarse de otro factor, a saber, de la jerarquización de estas restricciones. Las restricciones no son absolutas, de manera que todas pueden ser transgredidas varias veces, por lo que la jerarquización en cada variedad lingüística en particular es imperiosa para la determinación del candidato más óptimo.

Con respecto al funcionamiento de EVAL, esta función empieza con la restricción posicionada en el nivel más alto de la jerarquía. De aquí, extrae los candidatos que cometen la menor cantidad de violaciones y descarta todos los demás. Este proceso continúa en sentido descendente en la jerarquía, hasta que solo queda un candidato, el cual es el óptimo y, por lo tanto, el educto. En muchas ocasiones, todos los candidatos restantes desobedecen una restricción; en estos casos, los que producen la menor cantidad de transgresiones son los que prevalecen. Dada la gran cantidad de restricciones universales que tienen que existir, cualquier candidato, inclusive el ganador, siempre incumple muchas de estas. Sin embargo, la mayoría de las restricciones son inactivas, lo cual quiere decir que tienen menor rango que la restricción que elimina al último candidato no ganador.

Los análisis se suelen representar por medio de una tabla con características particulares. El aducto se presenta en la celda superior izquierda. Abajo, en la misma columna, se colocan todos los candidatos necesarios para ilustrar el análisis de manera clara. El candidato que resulta ser el óptimo se señala con una mano. A su vez, en la primera fila, se presenta la jerarquía de restricciones, solamente las relevantes para el análisis en cuestión, con la de mayor rango a la izquierda, de modo que cada restricción domina a las que se encuentran a su derecha. No obstante, es común que no existan evidencias de una priorización respectiva entre dos o más restricciones en una lengua; en tales casos, la división entre las columnas consiste en una 


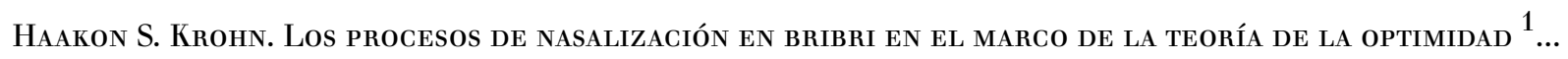

línea punteada, mientras que las líneas entre las restricciones cuya jerarquización sí se puede distinguir son continuas.

Los incumplimientos a las restricciones se marcan con asteriscos, uno por cada transgresión. Además, se utiliza un signo de exclamación a la derecha de las marcas que causan la eliminación de candidatos. Otra convención es un sombreado gris en las celdas correspondientes a las restricciones que son irrelevantes para un candidato en concreto, sea porque este ya ha sido eliminado en un nivel superior de la jerarquía o porque es el último candidato restante al nivel de esta restricción.

\subsection{Armonía nasal}

La armonía nasal es un patrón fonológico que consiste en una transmisión de nasalidad a larga distancia. Esta nasalidad proviene de un segmento fonológicamente nasal y es propagada a una serie de segmentos, contiguos o no, hasta que sea detenida. Al tratarse los diferentes segmentos involucrados en este fenómeno, se emplean los siguientes términos (Walker, 2011, p. 1):

- Desencadenante ("trigger"): un segmento fonológicamente nasal que desencadena la armonía nasal.

- Blanco ("target"): un segmento que se puede nasalizar mediante la armonía nasal.

- Bloqueador o segmento opaco: un segmento que no se puede nasalizar y detiene la armonía nasal.

- Segmento transparente: un segmento que no se puede nasalizar por medio de la armonía nasal, pero que no la detiene.

La propagación nasal por causa de armonía puede ocurrir hacia la derecha, hacia la izquierda o en ambas direcciones, dependiendo de las configuraciones fonológicas de la lengua en cuestión. Todos los segmentos blanco que se encuentren en la dirección determinada con respecto al desencadenante son nasalizados, a menos que intervenga uno de dos tipos de elementos que detienen la propagación: un segmento bloqueador o un linde del dominio de la armonía nasal. El dominio es típicamente la palabra o alguna unidad morfológica o prosódica menor (Walker, 2011, p. 1). Nuestro análisis se basará en el modelo fonológico autosegmental (Goldsmith, 1976a, 1976b), por lo que la transmisión de nasalidad será interpretada como asociaciones de segmentos fonológicamente no nasales con un mismo autosegmento [+nasal].

De acuerdo con Walker (1998, p. 24), el conjunto de segmentos bloqueadores en cada lengua suele concordar con la jerarquía de sonoridad, de forma que alguna secuencia ininterrumpida de fonemas en el extremo obstruyente de la jerarquía funcionan como bloqueadores. Este no es el caso en el bribri, puesto que las oclusivas sonoras son blancos, mientras que las fricativas, que son más sonorantes que las oclusivas, no se pueden nasalizar. Este patrón puede explicarse como una consecuencia de la dificultad asociada con la producción de obstruyentes nasales (Ohala y Solé, 2008, pp. 319-321), en combinación con el hecho de que las oclusivas sonoras en bribri pasan a ser sonorantes cuando se nasalizan. Sin embargo, también las vocales tónicas son bloqueadores, lo cual tiene que deberse a un principio de preservación de contrastes fonológicos en sílabas acentuadas.

\subsection{Nasalización por hipersonorización}

La hipersonorización ("hypervoicing") es un conjunto de estrategias para mantener la sonoridad de consonantes en posiciones periféricas (inicial y final absolutas). Según Ohala (2011, p. 64), la restricción aerodinámica de sonorización ("Aerodynamic Voicing Constraint", AVC), la cual dicta que se necesita una corriente de determinada cantidad de aire a través de las cuerdas vocales para que se logre pronunciar una consonante sonora, puede causar la desonorización de consonantes fonológicamente sonoras en dichas posiciones. Como una medida preventiva, interviene la hipersonorización para asegurar el mantenimiento 
de la sonoridad. Chevrier (2017, pp. 294-303) enumera una serie de estrategias de hipersonorización, tales como la retroflexión y el flapping (ambas presentes en el bribri), pero la que tiene relevancia para el presente estudio es la nasalización: las oclusivas sonoras en posición final de sílaba son nasalizadas en ciertas ocasiones para mantener su sonoridad.

\section{AsPectos RELEVANTES DE LA FONOLOGÍA BRIBRI}

\subsection{Inventario fonémico}

El bribri presenta contraste fonológico entre vocales orales y nasales. La serie oral consta de siete fonemas:

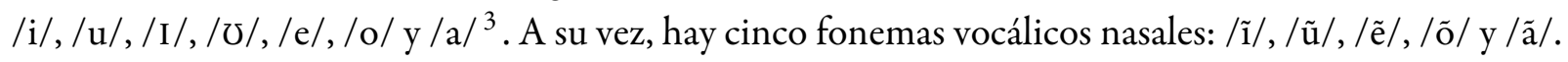

En cambio, la lengua no posee consonantes nasales a nivel fonológico, un hecho que puede considerarse una peculiaridad tipológica, como es señalado por Constenla (1985) y Chevrier (2017, pp. 144-145), ya que, de acuerdo con los datos examinados por Maddieson (2013), menos de un $2 \%$ de las lenguas del mundo carecen de consonantes fonológicamente nasales. Esta configuración parece haberse mantenido desde el protochibcha, para el cual Constenla $(2008,2012)$ y Pache $(2018)$ reconstruyen un sistema fonológico muy similar al del bribri, con contraste nasal en las vocales, pero no en las consonantes. Entre las lenguas chibchenses habladas en la actualidad, solo el bribri, el cabécar y, probablemente, el buglere y el naso (Chevrier, 2017, pp. 361-366) presentan esta configuración; en las demás han llegado a fonemizarse consonantes nasales, principalmente por influencia de la nasalidad vocálica, y muchas de las lenguas han perdido la serie de vocales nasales.

En la Tabla 1 se presentan los fonemas consonánticos del bribri, de acuerdo con el análisis de Chevrier (2017).

TABLA 1.

Fonemas consonánticos del bribri (Chevrier, 2017, p. 60)

\begin{tabular}{|l|c|c|c|c|c|c|c|c|}
\hline & \multicolumn{2}{|c|}{ Labial } & \multicolumn{2}{c|}{ Coronal } & \multicolumn{2}{c|}{ Palatal } & Velar & Glotal \\
\hline & Sorda & Sonora & Sorda & Sonora & Sorda & Sonora & Sorda & Sorda \\
\hline Oclusiva & $\mathrm{p}$ & $\mathrm{b}$ & $\mathrm{t}$ & $\mathrm{d}$ & $\mathrm{tc}$ & & $\mathrm{k}$ & $?$ \\
\hline Africada & & & $\overline{\mathrm{ts}}$ & & $\overline{\mathrm{t}}$ & $\overline{\mathrm{d}} \mathrm{s}$ & & \\
\hline Fricativa & & & $\mathrm{s}$ & & $\mathrm{\int}$ & & & $\mathrm{h}$ \\
\hline Vibrante & & & & $\mathrm{r}$ & & & & \\
\hline
\end{tabular}

Cabe mencionar que aquí hemos realizado algunas modificaciones con respecto a la tabla presentada por Chevrier (2017, p. 60). En primer lugar, hemos excluido las aproximantes (representadas por Chevrier como $/ w / y / j /$ ) del conjunto de fonemas consonánticos, ya que la distribución de [w] y [j] indica con bastante claridad que son alófonos no silábicos de las vocales /u/ e /i/, respectivamente. En segundo lugar, hemos empleado el símbolo / $/ \bar{d} /$ en lugar de / J/, dado que se trata de una africada. Además, hemos escogido

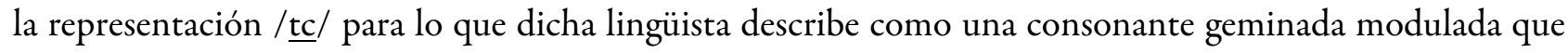

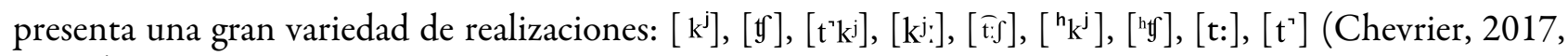
p. 444).

Aparte de algunas diferencias menores, el análisis de Chevrier (2017) se distingue de los presentados en otras obras importantes sobre el bribri por un hecho particular: la clasificación de la vibrante simple retrofleja 


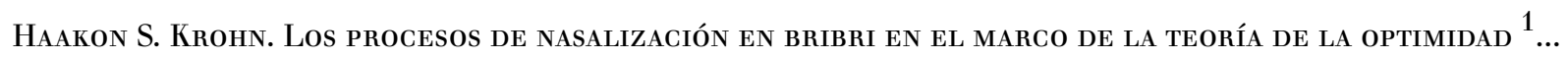

[r] (a menudo descrita como vibrante simple alveolar lateral [I] por otros autores ${ }^{4}$ ) como alófono de /d/; en contraste, Wilson (1982), Constenla, Elizondo y Pereira (1998) y Jara (2018) postulan un fonema /I/. A su vez, todos estos autores clasifican la vibrante simple alveolar $[r]$ como alófono de /d/, mientras que Chevrier (2017) la clasifica como la realización más común del fonema / r/. Asimismo, tanto Wilson (1982) como Jara (2018) postulan un fonema vibrante múltiple alveolar /r/, mientras que Constenla, Elizondo y Pereira (1998) consideran que un fonema de tales características sí se manifiesta, pero solo en algunos préstamos del español. En cambio, de acuerdo con Chevrier (2017), [r] es alófono de / r/. En el presente trabajo, partimos del análisis de Chevrier (2017) por el hecho de que es más riguroso que los otros, además de que es el único que se basa explícitamente en datos acústicos de espectrogramas.

\subsection{Estructura de las palabras}

A diferencia del análisis fonémico, el trabajo de Chevrier (2017) es menos preciso con respecto a la descripción de la estructura de las palabras bribris, por lo que aquí se proporcionará una descripción más detallada. En bribri, la última sílaba de todas las raíces léxicas, así como la de una considerable cantidad de palabras gramaticales, es acentuada. Esto quiere decir que la mayoría de las palabras presentan exactamente una sílaba con acento primario. Las excepciones son las palabras gramaticales con raíces átonas (las cuales, posiblemente, podrían analizarse fonológicamente como clíticos) y un conjunto de palabras (en su mayoría adjetivos, pero también de varias otras clases) que contienen una raíz tónica reduplicada (aunque parece que el último de estos dos acentos se encuentra en proceso de neutralización en muchos casos). También es posible que las sílabas postónicas deban considerarse como portadores de acento secundario, puesto que presentan contrastes entre todos los fonemas vocálicos y pueden contener una coda, al igual que las sílabas con acento primario. De todos modos, esto tiene que investigarse con mayor detalle, por lo que utilizaremos de aquí en adelante el término sílaba inacentuadalátona para todas las sílabas sin acento primario, mientras que sílaba acentuada/tónica solo aludirá al acento primario.

Aunque las descripciones existentes de la fonología bribri solo hacen la distinción entre sílabas acentuadas (o fuertes) e inacentuadas (o débiles), la característica de las sílabas postónicas que se acaba de mencionar significa que la palabra bribri puede, en realidad, dividirse en tres partes: sílabas pretónicas, sílaba tónica y sílabas postónicas. Las sílabas tónicas son las únicas que presentan un tonema fonológicamente contrastivo, lo cual quiere decir que el bribri puede denominarse una lengua de acento tonal contrastivo ${ }^{5}$, como indicado por Wilson (1982, p. xxiii). Los tonos contrastivos son, por lo menos, dos: alto/ascendente y descendente. Según Constenla, Elizondo y Pereira (1998, p. xi), el dialecto de Amubre presenta un tercer tono contrastivo, descrito como ascendente, pero no proporcionan ningún par mínimo que justifique la presunta oposición fonológica entre los tonos alto y ascendente; por lo tanto, es probable que se trate de dos alótonos del mismo tonema ${ }^{6}$. En este artículo, no se hará ninguna diferenciación entre estos dos tonos, y todas las instancias correspondientes se transcribirán con el diacrítico de tono alto.

En términos de la fonología autosegmental, las sílabas de tono alto están asociadas con el elemento tonal $\mathrm{H}$ (alto), mientras que las de tono descendente se vinculan con una secuencia de dos tonos, $\mathrm{H}$ y $\mathrm{L}$ (alto y bajo). En contraste, todas las sílabas inacentuadas se pronuncian con tono bajo, o neutro, el cual debe considerarse el tono por defecto. Por consiguiente, una propiedad que distingue a todas las sílabas acentuadas de las inacentuadas es su asociación con un autosegmento $\mathrm{H}$.

En lo que concierne al nivel segmental, tanto en las sílabas tónicas como postónicas aparecen, de manera contrastiva, todas las calidades vocálicas fonológicas, así como la oposición entre vocales orales y nasales. La mayoría de las sílabas inacentuadas en bribri se encuentran en posición postónica, puesto que, con la excepción de una serie de prefijos de persona, todos los afijos son sufijos. Muchos de estos sufijos todavía conservan todos los segmentos, de manera inalterada, de las palabras léxicas a partir de las cuales fueron 
gramaticalizados, pero su tono se encuentra neutralizado debido a que han perdido el acento léxico primario. Por añadidura, en palabras compuestas por dos sustantivos, el tono de la raíz del segundo sustantivo se halla neutralizado, por lo que está claro que la composición léxica sigue los mismos patrones fonológicos que la gramaticalización de los sufijos.

Las sílabas pretónicas son las que más se distinguen de las tónicas. Además de siempre ser pronunciadas con tono bajo y nunca tener coda silábica, presentan una neutralización vocálica muy fuerte: sus vocales son breves y de baja intensidad, pueden nasalizarse, centralizarse hacia [ə], asimilarse a la calidad de la vocal de la sílaba siguiente e incluso elidirse (Chevrier, 2017, p. 211). Según Chevrier (2017, p. 100), quien no distingue entre sílabas pretónicas y postónicas, los núcleos de sílabas inacentuadas (entiéndase como sílabas prétonicas) están subespecificadas, lo cual explicaría su alto grado de variabilidad. Cuando no hay reducción ni aparente influencia por otra vocal, las vocales pretónicas se manifiestan como [i], [a] o [u] (Chevrier, 2017, p. 101), pero no se ha encontrado ningún par mínimo con contraste entre vocales pretónicas. Asimismo, está completamente claro que la nasalidad no es contrastiva en esta posición.

\section{Procesos de nasalización en bribri}

El presente análisis se fundamenta principalmente en los datos presentados en la investigación tipológica de Chevrier (2017), la descripción más rigurosa que existe acerca de la nasalización en bribri. Dicha lingüista analiza solamente dos de los tres dialectos principales de esta lengua, a saber, el de Amubre y el de Coroma, ambos hablados en el Valle de Talamanca en la vertiente del océano Atlántico. Por esta razón, no se considerarán fenómenos de nasalización en el dialecto de Salitre, hablado en la vertiente del océano Pacífico; no obstante, hay vestigios de que los patrones de nasalización en esta variedad son muy parecidos a los del bribri de Amubre (Chevrier, 2017, pp. 237-238).

La nasalización de segmentos que a nivel fonológico no son nasales en bribri es un fenómeno bien conocido que se señala en todas las descripciones fonológicas generales de esta lengua (Wilson, 1974, 1982; Schlabach, 1974; Constenla, 1981, pp. 106-117, 179-180; Constenla, Elizondo y Pereira, 1998; Jara, 2018). Además, ha sido objeto de estudio específico de varias investigaciones (Wilson, 1970; Constenla, 1982, 1985; Tohsaku, 1987; Chevrier, 2017, pp. 139-351). Los distintos procesos de nasalización identificados por Chevrier (2017) se detallan a continuación.

El proceso de nasalización que se da con mayor frecuencia en bribri es la armonía manifestada como propagación nasal hacia la izquierda. Esta funciona de la misma manera en los dos dialectos analizados y consiste en que todas las vocales átonas (incluidos sus alófonos aproximantes) y todas las consonantes sonoras -/b/,/d/, / / $\overline{\mathrm{d}} / \mathrm{y} / \mathrm{r} /$ - se nasalizan cuando se hallan ante un sonido nasal. Chevrier (2017, pp. 249-251) afirma que estos fonemas están subespecificados para el rasgo [nasal]. Es importante enfatizar que un segmento que fonológicamente no es nasal, pero que ha sido nasalizado por medio de este proceso, también nasaliza al segmento que se encuentra a su izquierda en caso de que este también sea uno de los fonemas nasalizables, por lo que una vocal fonológicamente nasal puede transferir el rasgo [+nasal] a una cadena de segmentos. No obstante, la propagación siempre es detenida por las vocales orales acentuadas y las consonantes sordas, elementos bloqueadores que no son afectados por la propagación nasal. Además, los lindes de palabra también detienen la propagación. El alófono nasal de /b/ es $[\mathrm{m}]$, el de $/ \mathrm{r} /$ es $[\mathrm{n}]$ y el de $/ \widehat{\mathrm{d}_{3}} / \mathrm{es}[\mathrm{n}]$. En cuanto a $/ \mathrm{d} /$, también presenta el alófono nasal [n], excepto en posición interior de morfema, en la que su realización nasal

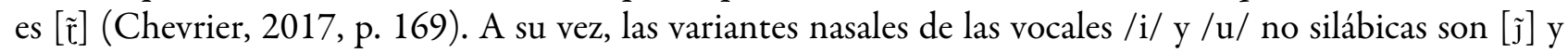
$[\tilde{\mathrm{w}}]$, respectivamente.

A continuación, se ofrecen varios ejemplos de este fenómeno. Para no embarullar la interpretación de los datos, todas las formas fonéticas corresponden a un habla muy cuidada, sin elisión ni reducción vocálica en sílabas átonas. Además, como otra medida para evitar complicaciones innecesarias, los núcleos silábicos pretónicos son representados fonológicamente como /a/ en todos los casos, aunque, como se explicó 


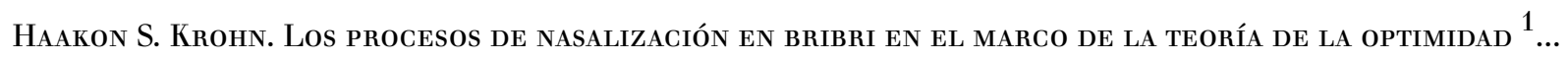

anteriormente, puede ser que las vocales en esta posición estén subespecificadas. De todos modos, dichas mesuras no afectan el análisis de la nasalización, la cual no es alterada por vocales reducidas o elididas. Asimismo, muchas de las formas bribris expuestas presentan variaciones dialectales no incluidas aquí, lo cual tampoco afecta el análisis de ninguna manera.

Para empezar ${ }^{7}$, en los ejemplos 1-6 se observan procesos de propagación que llegan hasta el extremo izquierdo de la palabra (el sonido [r] en el primer ejemplo es alófono de /d/ en posición final de palabra); además, en estas palabras se pueden apreciar los alófonos nasales de los seis fonemas nasalizables.

(1) /bađ̧̧ẫd/ [mãnẫ] 'tres (ref. a seres humanos u objetos esféricos)'

(2) /dabấ/ [nãmấ] 'pez (gen.)’

(3) /đ̧̧adấ/ [nã̃ấa 'camino'

(4) /adề/ [ã̃̂ิ̂ิ] 'taltuza'

(5) /iỗk/ [jô̂k] 'gavilán aludo'

(6) /auî̃/ [ãwîi] 'aquel, aquello'

A su vez, en 7-12 se aprecia que las consonantes sordas no se nasalizan.

(7) /pû// [pû̃] 'ave rapaz (gen.)'

(8) /tû̃rũk/ [tûnũk] 'correr'

(9) /karế/ [kãnế] 'trabajo, labor'

(10) /țarê// [ţãnê] 'costilla'

(11) /sirấ/ [sĩnấ] 'oso perezoso'

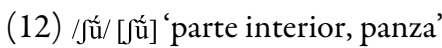

Los ejemplos 13-15 ilustran mejor el funcionamiento de los bloqueadores: las consonantes sordas se mantienen orales y detienen la propagación de la nasalidad. Aquí cabe resaltar que las fricativas sordas -/s/, / / / y /h/ - no se han podido observar en posición no inicial ante un segmento nasal, por lo que no hay manera de constatar si son bloqueadores o transparentes. De todos modos, se etiquetan como bloqueadores en el presente artículo, ya que no existe ninguna indicación de lo contrario, pero esto no influye en el análisis.

(13) /batấ/ [batấ] 'ardilla'

(14) /kakố/ [kakố] 'tortuga baula'

(15) /batsú́/ [batsû́] 'colibri'

En los ejemplos 16-17 se muestra otro tipo de bloqueador, las vocales tónicas: hay una vocal oral acentuada ante consonante nasal que se mantiene oral y, a la vez, detiene la propagación.

(16) /ḑ̧ábĩ/ [đ̧zámĩ] 'pariente'

(17) /iárũk/ [jánũk] 'ensuciarse’

Por último, en 18-19 se observa que incluso una vocal oral que se encuentra inmediatamente ante una vocal fonológicamente nasal se mantiene oral.

(18) /adíũk/ [ariũu] 'madurar'

(19) /diỗẽ/ [djỗe] 'allá' 
El segundo tipo de nasalización tiene lugar en la coda silábica e involucra los fonemas sonoros /b/, $/ \mathrm{d} / \mathrm{y} / \mathrm{r} /(/ \widehat{\mathrm{d}} / \mathrm{h}$ no ocurre en esta posición). Como ya se ha mencionado, solamente las sílabas tónicas $\mathrm{y}$ postónicas pueden tener coda en bribri, y estas codas siempre se encuentran al final de un morfema. En ambos dialectos bajo estudio, el fonema /b/ en esta posición se puede nasalizar, independientemente de si la vocal precedente sea oral o nasal; esto es obligatorio en todos los casos en Coroma, mientras que se observa una variación libre entre $[\mathrm{m}],[\mathrm{b}]$ y [ $\mathrm{mb}]$ en Amubre, excepto en posición final de enunciado, donde solo se da $[\mathrm{m}]$ (Chevrier, 2017, pp. 272-275) ${ }^{8}$. A su vez, la realización de /d/ y / r/ presenta aún más variación dialectal (Chevrier, 2017, pp. 278-282): en Amubre se nasalizan si la vocal precedente es nasal y pertenece al mismo morfema que la consonante, mientras que en Coroma no se nasalizan en coda silábica, excepto en los casos de reduplicación, que se tratarán más adelante. Chevrier (2017) interpreta la nasalización después de vocal nasal como nasalización progresiva, y la nasalización de /b/ sin entorno nasal como hipersonorización.

Añadido a esto, Chevrier (2017, p. 270) observa más detalles acerca de los fonemas /b/y /d/ en la variedad de Amubre: /d/ también se realiza como nasal en posición final de enunciado (ante pausa), y tanto /b/ como $/ \mathrm{d} /$ presentan alófonos orales y nasales en variación libre en posición final de morfema en el interior de enunciados. Asimismo, /b/ presenta un alófono [ $\mathrm{y}]$ que se encuentra en variación libre con $[\mathrm{m}]$ en ambos dialectos (Chevrier, 2017, p. 277). Estos datos indudablemente son relevantes, pero no se tomarán en cuenta en el análisis teórico-óptimo, puesto que solo constituyen variaciones mínimas de fenómenos similares.

En los ejemplos 20-21, presentados a continuación, la /b/ final es nasalizada independientemente de la característica de la vocal precedente, fenómeno que está presente en ambos dialectos analizados.

(20) /dabób/[dabóm] 'diez'

(21) /kadếb/ [kãấm] 'pez barbudo'

En cambio, la nasalización progresiva a /d/ y / $/$ / en coda silábica ocurre solamente en el dialecto de Amubre. El ejemplo 22 muestra que el fonema /d/ no se nasaliza cuando le precede una vocal oral, caso en el que se realiza como [r]. Lo mismo sucede cuando la consonante en cuestión no forma parte del mismo morfema que la vocal precedente, incluso si esta vocal es nasal, como se observa en el ejemplo 23, donde /-d/ es un clasificador numeral que alude a referentes humanos. En cambio, el fonema /d/ sí se nasaliza en posición de coda detrás de una vocal nasal que forma parte del mismo morfema, como en el ejemplo 24.

(22) /kád/[kár] 'árbol (gen.)’

(23) /skê̂+d/ [skê̂r] 'cinco (ref. a seres humanos)'

(24) /batsû́d/ [batsû́n] 'higuerón'

El fonema / $\mathrm{r}$ / sigue exactamente el mismo patrón, como se aprecia en los siguientes ejemplos; el 25 muestra la realización oral de la consonante final detrás de una vocal oral, mientras que el 26 ejemplifica la nasalización progresiva a partir de una vocal nasal en el mismo morfema.

(25) /ás+ir/ [ásic] 'barranco, precipicio, derrumbe'

(26) /tễr/ [tễn] 'metido, clavado'

La alofonía descrita hasta ahora es sintetizada de manera muy clara mediante una tabla elaborada por Chevrier (2017, p. 169). Esta información se reproduce a continuación en la Tabla 2, donde se exponen los alófonos de los cuatro fonemas consonánticos sonoros en distintos entornos ${ }^{9}$. La última columna está dividida en dos para los fonemas / $\mathrm{d} / \mathrm{y} / \mathrm{r} /$, donde el alófono a la izquierda corresponde al dialecto de Amubre (cuando el fonema forma parte del mismo morfema que la vocal), mientras que el de la derecha corresponde al dialecto de Coroma (y los casos en los que el fonema en cuestión constituye un sufijo monofonémico en el 


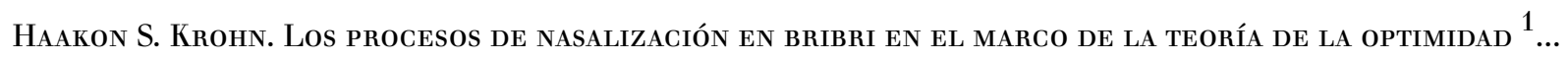

dialecto de Amubre). La tilde (“ ") que aparece en dos de las celdas indica variación libre en ambos dialectos, mientras que el guion (“-”) señala los entornos en los que nunca aparece el fonema en cuestión.

TABLA 2.

Alófonos de las consonantes sonoras en bribri (Chevrier, 2017, p. 169)

\begin{tabular}{|c|c|c|c|c|c|c|c|}
\hline & \multicolumn{2}{|c|}{$\begin{array}{c}\text { Ataque silábico, } \\
\text { inicio de morfema }\end{array}$} & \multicolumn{2}{|c|}{$\begin{array}{l}\text { Ataque silábico, } \\
\text { medio de morfema }\end{array}$} & \multicolumn{3}{|c|}{ Coda silábica } \\
\hline & $+\mathbf{V}$ & $+\tilde{\mathbf{V}}$ & $\mathbf{V}$-V & $\tilde{\mathbf{V}}_{-} \tilde{\mathbf{V}}$ & $\mathbf{v}_{-}+$ & \multicolumn{2}{|c|}{$\tilde{\mathbf{v}}_{-}^{+}$} \\
\hline$/ \mathbf{b} /$ & $\mathrm{b}$ & $\mathrm{m}$ & $\mathrm{b}$ & $\mathrm{m}$ & $\mathrm{m}$ & \multicolumn{2}{|c|}{$\mathrm{m}$} \\
\hline$/ \mathbf{d} /$ & d & $\mathrm{n}$ & $\mathrm{r}$ & $\tilde{\mathrm{r}}$ & $\mathrm{r}$ & $\mathrm{n}$ & $\mathrm{r}$ \\
\hline$/ \widehat{\mathbf{d}} \mathbf{3} /$ & $\overline{d_{3}}$ & $\mathrm{n}$ & $\widehat{d z}$ & $\mathrm{n}$ & - & \multicolumn{2}{|c|}{-} \\
\hline$/ \mathbf{r} /$ & - & - & $\mathrm{r} \sim \mathrm{r}$ & $\mathrm{n}$ & $\mathrm{r} \sim \mathrm{r}$ & $\mathrm{n}$ & f \\
\hline
\end{tabular}

La nasalización por hipersonorización en coda silábica también sucede en el dialecto de Amubre en palabras originadas por medio de la reduplicación de una raíz. A este respecto, es necesario ahondar un poco en la naturaleza de estos vocablos. El bribri tiene una considerable cantidad de palabras que claramente son el resultado de reduplicación morfológica, pero la reduplicación ya no es un recurso productivo en esta lengua, y parece que los hablantes generalmente no asocian el significado de la forma reduplicada con la de la raíz simple (en caso de que esta aparezca por sí sola), excepto, desde luego, en los casos en los que su semántica se ha mantenido muy similar. Por lo tanto, todo indica que, a nivel sincrónico, estas formas no son reduplicaciones, sino han sido consolidadas como bases semánticas sencillas. En consecuencia, las formas fonológicas deben incluir las raíces originales dos veces, pues ningún proceso que actualmente esté vigente en la lengua podría derivar las reduplicaciones a partir de una raíz sencilla.

En la formación de estas palabras, la primera consonante de la raíz se llegó a geminar al principio de la segunda instancia de la raíz. Cuando la consonante geminada es /b/ o /d/, el primero de los dos fonemas idénticos sufre un proceso fonológico: en el dialecto de Amubre se nasaliza, mientras que en Coroma se realiza como oclusiva glotal (Chevrier, 2017, p. 321). La nasalización que se da en Amubre se ilustra en los ejemplos 27 y 28, que incluyen datos de Chevrier (2017, pp. 287-289); las raíces sencillas de las que históricamente derivan son /bâ/ 'caliente' y / dí?/ 'agua', respectivamente.

(27) /bâbbá/ [bâmbá]'caliente'

(28) /díddí/ [díndí] 'afilado'

Antes de concluir este apartado, se puede agregar que Chevrier (2017, pp. 289-290) también observa algunos casos de "nasalización espontánea" de consonantes sordas en posición final de sílaba ante consonante sonora. No obstante, esto no es obligatorio, por lo que dicho fenómeno no se tomará en cuenta en nuestro análisis.

\section{ANÁLISIS}

El análisis teórico-óptimo de la nasalización en bribri se centrará primero la propagación nasal regresiva de los fonemas vocálicos nasales. A lo largo de todo el análisis, serán relevantes las dos restricciones de fidelidad fundamentales, las cuales se definen de la siguiente manera ${ }^{10}$ : 
$\operatorname{Max}$

Asignar una marca de transgresión por cada segmento en el aducto que no tenga un segmento correspondiente en el educto.

Dep

Asignar una marca de transgresión por cada segmento en el educto que no tenga un segmento correspondiente en el aducto.

Dicho de otro modo, MAX penaliza la elisión de segmentos y DEP penaliza la inserción de segmentos. Además, como ya se ha señalado, las vocales acentuadas en bribri siempre mantienen los rasgos de su forma fonológica, con respecto tanto a la calidad como a la nasalidad. Por consiguiente, proponemos una restricción de fidelidad, IDENT-V́, responsable de preservar todos los rasgos de las vocales acentuadas. Debido a que, a nivel interlingüístico, es común la reducción de vocales átonas y que, por ende, tiende a haber mayor cantidad de contrastes vocálicos en sílabas acentuadas que en sílabas inacentuadas (Gussenhoven, 2004, p. 15), la universalidad de esta restricción no parece controvertida:

\section{IDENT- V́}

Asignar una marca de transgresión por cada vocal acentuada en el aducto cuyo segmento correspondiente en el educto no presente los mismos valores para todos los rasgos.

Puesto que los segmentos bloqueadores en lenguas que presentan propagación nasal típicamente corresponden a una porción del extremo obstruyente de la jerarquía de sonoridad, es común que se utilicen restricciones con referencia a esta jerarquía para prevenir la nasalización de segmentos bloqueadores: ${ }^{*}$ NASPLO $>>{ }^{*}$ NASFRIC $>>{ }^{*}$ NASLIQ $>>{ }^{*}$ NASGLI $>>{ }^{*}$ NASVOW (Walker, 1998, p. 36; 2011, p. 19). No obstante, como los bloqueadores en bribri no corresponden a una secuencia continua en la jerarquía de sonoridad, ninguna de estas restricciones puede encontrarse activa en esta lengua. En cambio, es necesario hacer uso de una restricción de marcadez que penalice segmentos sordos nasales: *NAS/SOR. Esta se define de la siguiente manera:

\section{${ }^{*}$ NAS/SOR}

Asignar una marca de transgresión por cada segmento nasal sordo.

Asimismo, para evitar que un fonema sordo se sonorice para nasalizarse de manera subsecuente, la identidad del rasgo [sonoro] de los segmentos en el aducto debe preservarse, lo cual será asegurado por medio de la restricción de fidelidad IDENT([son]):

\section{$\operatorname{IDENT}([$ son $])$}

Asignar una marca de transgresión por cada segmento en el educto que difiera del segmento correspondiente en el aducto en cuanto al valor del rasgo [sonoro].

La propagación nasal ha sido, y sigue siendo, un reto para la teoría de la optimidad. Una solución intuitiva sería una restricción que prohíba dos segmentos contiguos con valores distintos para el rasgo [nasal]: AGREEL(nas) para la propagación hacia la izquierda y AGREE-R(nas) para la dirección opuesta. No obstante, como muestran Wilson $(2003,2004,2006)$ y McCarthy $(2004,2008,2011)$, tal aproximación no funciona, debido a que desfavorece la propagación nasal cuando esta no puede llegar hasta el extremo de su dominio.

Por lo tanto, en el presente análisis hacemos uso de una restricción de alineamiento a larga distancia, de la familia ALIGN, comúnmente empleada para describir el fenómeno en cuestión (véase McCarthy, 2008, p. 231; 2011, p. 5). Aunque Wilson (2003, 2004, 2006) y McCarthy (2004, 2008, 2011) también han encontrado problemas relacionados con esta aproximación, en el sentido de que predice que la elisión de segmentos que se encuentran al otro lado de un elemento bloqueador debería ser común en las lenguas con propagación de rasgos, esto nunca produce eductos erróneos. Otras alternativas propuestas, como el serialismo harmónico ("Harmonic Serialism”) de McCarthy (2011, 2016), alteran sustancialmente el funcionamiento de GEN, un componente fundamental de la teoría de la optimidad. En el presente análisis, 


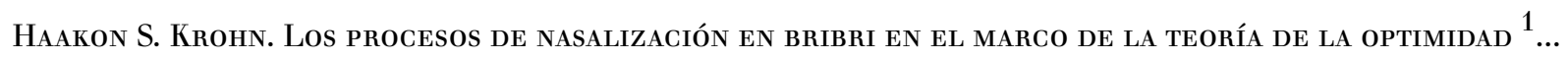

deseamos apegarnos lo más posible a la versión estándar de la teoría, por lo que consideramos que el alineamiento es la mejor opción.

La instancia de ALIGN que cataliza la propagación nasal en bribri la llamaremos ALIN-I([+nas], pal) (como abreviación de Alinear-Izquierda) en español, y se define del siguiente modo:

ALIN-I([+nas], pal)

Asignar una marca de transgresión por cada segmento entre un segmento nasal y el linde izquierdo de una palabra que no esté asociado con el mismo autosegmento [+nasal].

Esta definición se basa en la representación de la fonología autosegmental, según la cual [+nasal] es un autosegmento que puede estar asociado con varios segmentos simultáneamente. La dirección del alineamiento motivado por esta restricción es hacia la izquierda, [+nas] es el rasgo propagado y "pal" es una abreviación de "palabra", lo cual quiere decir que el dominio de la propagación es la palabra, cuyos lindes, por tanto, detienen el proceso. Se postula la presencia de un autosegmento [+nasal] por cada vocal fonológicamente nasal, y que todos los fonemas afectados por la armonía nasal son asociados al mismo autosegmento [+ nasal] que es responsable de la propagación.

Para ejemplificar la asignación de marcas de transgresión producidas por esta restricción, en el caso de una palabra en la que la propagación llega hasta el extremo izquierdo, tal como /bađ̧̧âd/ [mãnẫ] 'tres', no se asignaría ninguna marca, pues los tres fonemas que se hallan entre el linde izquierdo de la palabra y la vocal fonológicamente nasal, /ã/, están asociados con el mismo autosegmento [+ nasal] que /ã/. A su vez, en un caso como /batsúf/ [batsû]] 'colibrí', se asignarían tres marcas, porque tres fonemas a la izquierda de la vocal nasal, / ũ/, no se encuentran asociados con [+nasal]. Por añadidura, es importante señalar que la restricción implica que todos los fonemas asociados con el mismo autosegmento sean contiguos; en caso de que no, estarían presentes dos autosegmentos [+nasal] diferentes. Por ejemplo, si se diera una nasalización espontánea del primer fonema de /batsû́/, es decir, [matsúu, también se asignarían tres marcas de violación, porque /b/ [m] no estaría vinculado con el mismo autosegmento que / ũ/, de manera que todavía habría una distancia de tres segmentos entre el rasgo [+ nasal] de ese último y el linde izquierdo de la palabra.

Aunque las realizaciones con nasalizaciones espontáneas del tipo ejemplificado no reducen la cantidad de marcas de transgresión, hace falta una restricción para eliminar estos candidatos, ya que tal fenómeno no ocurre en bribri. Para este propósito, se necesita una restricción de fidelidad, $\mathrm{DEP}([+$ nas $])$, que penaliza cada nasalización:

\section{$\operatorname{DEP}([+$ nas $])$}

Asignar una marca de transgresión por cada segmento nasal en el educto cuyo segmento correspondiente en el aducto no sea nasal.

Cabe mencionar que también tiene que estar involucrada una serie de restricciones que aseguren la correspondencia entre el aducto y el educto con respecto a otros rasgos, a saber, los que especifican el punto y el modo (con la excepción de [nasal]) de articulación. Sin embargo, estos no se incluirán en los análisis, ya que quedan fuera del alcance del presente estudio.

Con estas restricciones definidas, es hora de indagar en su ordenamiento respectivo en el bribri. En primer lugar, está claro que las restricciones que nunca son violadas tienen que ubicarse en el nivel superior, y que no pueden ser ordenadas recíprocamente (McCarthy, 2008, p. 65). Este es el caso de DEP, ya que no se han observado inserciones en el bribri, y de IDENT-V́, pues las vocales tónicas siempre conservan su calidad y su valor de nasalidad.

En cuanto a MAX, sí ocurren elisiones de vocales pretónicas, como ya se ha señalado, por lo que esta restricción en realidad debe hallarse en un nivel inferior, dominada por alguna restricción que asegure que se elidan las vocales cuando corresponda ${ }^{11}$. No obstante, las elisiones no son relevantes para la nasalización y no proveemos ninguna prueba del ordenamiento de MAX en los ejemplos proporcionados en este artículo, por lo que se colocará al mismo nivel que DEP e IDENT-V́ en las tablas. 
Con respecto a IDENT([son]), sí se observan en el bribri varios casos de desonorización de consonantes ante consonante sorda como resultado de elisiones vocálicas, así como una sonorización esporádica de / $\mathrm{k} /$ (Chevrier, 2017, p. 269). Sin embargo, al igual que el caso MAX, estos procesos no son relevantes para los fenómenos analizados ni aparecen en los ejemplos presentados a lo largo del escrito, por lo que también esta restricción se colocará al mismo nivel que las anteriores.

En cambio, ALIN-I([+nas], pal) y DEP([+nas]) son frecuentemente transgredidas, debido a los segmentos bloqueadores y las nasalizaciones, respectivamente. Por consiguiente, estas tienen que estar dominadas por las demás restricciones mencionadas. Asimismo, como la obediencia a ALIN-I([+nas], pal) siempre causará violaciones a $\operatorname{DEP}([+$ nas $])$ y las nasalizaciones de fonemas nasalizables son preferidas dentro del dominio de propagación, ALIN-I $([+$ nas $]$, pal $)$ tiene que dominar a $\mathrm{DEP}([+$ nas $])$.

La interacción entre las restricciones en una palabra en la que la nasalidad de una vocal se propaga hasta el extremo izquierdo, a saber, /dabấ/ [nãấ] 'pez', se ilustra en la Tabla 3. Para simplificar la presentación, aquí no se incluyen ${ }^{*} \mathrm{NAS} / \mathrm{SOR}$ e IDENT([son]), que son inactivas debido a que la palabra no contiene ningún fonema sordo, ni DEP, pues una inserción tampoco prevendría ninguna violación a las otras restricciones.

TABLA 3.

Análisis de la palabra /dabấ/ 'pez'

\begin{tabular}{|c|c|c|c|c|c|}
\hline & /dabã̃/ & IDENT-V' & MAX & $\begin{array}{c}\text { ALIN-I } \\
(\lceil+ \text { nas }\rceil, \text { pal })\end{array}$ & $\operatorname{DEP}([+$ nas $])$ \\
\hline a. & nãmã́ & & & & $* * *$ \\
\hline b. & dãmã̃ & & & $* !$ & $* *$ \\
\hline c. & damã̃ & & & $* ! *$ & * \\
\hline d. & dabấ & & & $* ! * *$ & \\
\hline e. & dabá & $* !$ & & & \\
\hline f. & dab & $* !$ & $* !$ & & \\
\hline g. & ãmấ & & $* !$ & & $* *$ \\
\hline h. & nabấ & & & $* ! * *$ & $*$ \\
\hline
\end{tabular}

Se puede observar que las dos restricciones superiores en la jerarquía, IDENT-V́ y MAX, previenen las alteraciones a la vocal acentuada y las elisiones en los candidatos $e-g$, los cuales, en caso contrario, le ganarían al candidato $a$. Asimismo, debido a que cada incumplimiento con ALIN-I([+nas], pal) provoca una marca de transgresión, [nãmá] sale ganando frente a los candidatos $b-d$, ya que todos los segmentos están asociados con el mismo autosegmento [+nasal]. El candidato $b$ está incluido en la tabla para ilustrar que la nasalización espontánea, en este caso de /d/, no reduce la cantidad de violaciones a ALIN-I([+nas], pal).

Ahora se indagará en una palabra que contiene un bloqueador: /batấ/, [batấ] 'ardilla'. En este análisis, ilustrado en la Tabla 4, se incluyen *NAS/SOR e IDENT([son]) para mostrar cómo estas previenen la nasalización de fonemas sordos, pero se excluye MAX. 


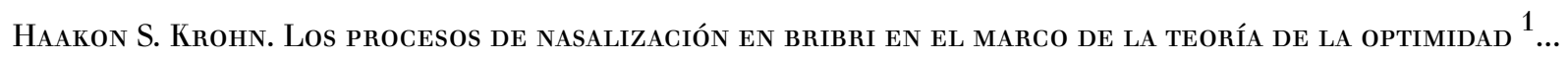

TABLA 4.

Análisis de la palabra /batấ/ 'ardilla'

\begin{tabular}{|c|c|c|c|c|c|c|}
\hline & /batấ/ & IDENT-V' & *NAS/SOR & $\operatorname{IDENT}([$ son $])$ & $\begin{array}{c}\text { ALIN- } \\
\mathrm{I}(\lceil+ \text { nas }\rceil, \text { pal })\end{array}$ & $\operatorname{DEP}([+$ nas $])$ \\
\hline a. $\sigma$ & batấ & & & & $* * *$ & \\
\hline b. & batá & $* !$ & & & & \\
\hline c. & mãnã & & $* !$ & & & $* * *$ \\
\hline d. & mãnã & & & $* !$ & & $* * *$ \\
\hline e. & matã́ & & & & $* * *$ & $* !$ \\
\hline
\end{tabular}

En este caso, la consonante / $t$ / bloquea la propagación nasal como consecuencia de dos restricciones: ${ }^{*}$ NAS/SOR penaliza la nasalización de dicho segmento, por lo que se le asigna una marca fatal al candidato $c$, mientras que IDENT([son]) penaliza su sonorización, de manera que no se puede nasalizar subsecuentemente, como en $d$. Dado que el segmento opaco se encuentra inmediatamente a la izquierda de la vocal fonológicamente nasal, el candidato ganador, $a$, y el candidato con nasalización espontánea del primer segmento, $e$, reciben la misma cantidad de marcas para la restricción de alineamiento, de ahí que $\mathrm{DEP}([+$ nas $])$ sea crucial para inhibir la nasalización espontánea. Además, al igual que en el análisis anterior, IDENT-V́ previene la desnalización de /ã/, como en el candidato $b$.

Otro tipo de elemento bloqueador son las vocales orales acentuadas, así que corresponde presentar un ejemplo de este también: específicamente, se analizará la palabra /đđđábìi [đ̧̧ámĩ] 'pariente'. Este vocablo contiene una vocal fonológicamente nasal átona, por lo que la restricción IDENT-V́ no prevendría la elisión de esta nasalidad, a menos que se postule un acento secundario en el sufijo /-bĩ/, lo cual, ciertamente, podría ser el análisis más apropiado. Sin embargo, en caso de que dicha suposición no resulte correcta, se propone aquí una restricción adicional que daría cuenta de la conservación de la nasalidad en vocales postónicas:

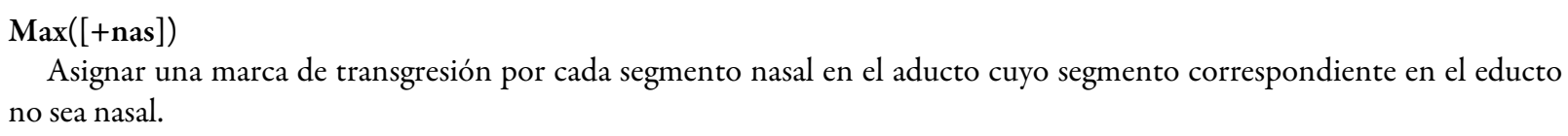

Asignar una marca de transgresión por cada segmento nasal en el aducto cuyo segmento correspondiente en el educto no sea nasal.

Dicho de otra manera, esta restricción penaliza cualquier desnasalización, independientemente de si la vocal en cuestión lleve acento o no. Ya que esta restricción nunca es transgredida, se ubica al primer nivel en la jerarquía. La interacción entre las restricciones para la palabra /đ̧ábĩ/ [đ̧ámî] 'pariente' se muestra en la Tabla 5, donde nuevamente se excluyen ${ }^{*}$ NAS/SOR e IDENT([son]). 
TABLA 5 .

Análisis de la palabra / ḑábĩ / 'pariente'

\begin{tabular}{|c|c|c|c|c|c|}
\hline & /ḑábĩ/ & IDENT-V' & $\operatorname{MAx}([+\mathrm{nas}])$ & $\begin{array}{c}\text { ALIN-I }([+ \text { nas }] \\
\text { pal })\end{array}$ & $\operatorname{DEP}([+$ nas $])$ \\
\hline a. & ḑ̧ámĩ & & & $* *$ & $*$ \\
\hline b. & ḑábĩ & & & $* * * !$ & \\
\hline c. & đ̧zấmĩ & $* !$ & & $*$ & $* *$ \\
\hline d. & jấmĩ & $* !$ & & & $* * *$ \\
\hline e. & dzábi & & $* !$ & & \\
\hline f. & jámĩ & & & $* *$ & $* * !$ \\
\hline
\end{tabular}

El candidato $b$ es eliminado porque un segmento nasalizable a la izquierda de la vocal nasal es oral, mientras que los candidatos $c-d$ pierden debido a la nasalización de la vocal oral acentuada, que es el elemento bloqueador en esta palabra. Por su lado, $\operatorname{MAX}([+$ nas $])$ se encarga de eliminar el candidato $e$, en el cual se desnasaliza la última vocal. Por último, el candidato $f$ es el perdedor que más perdura, pero la nasalización $\mathrm{de} / \widehat{\mathrm{d}_{3}} /$ es penalizada por $\mathrm{DEP}([+$ nas $])$. En síntesis, ALIN-I $([+$ nas $]$, pal $)$ es responsable de que el fonema /b/ se nasalice, mientras que $\mathrm{DEP}([+$ nas $])$ previene cualquier nasalización a la izquierda del bloqueador.

El siguiente proceso por analizar es la nasalización de /b/ en posición final de sílaba, el cual ocurre en ambos dialectos tratados y es explicado por Chevrier (2017) como un caso de hipersonorización ${ }^{12}$. Ya que el fenómeno de nasalización es el que interesa aquí, se ignorarán los casos de variación libre en el bribri de Amubre. Debido a que / $b$ / es la única consonante que se nasaliza en esta posición en el dialecto de Coroma, es inevitable postular una restricción muy específica que prohíba solo esta consonante, o posiblemente una clase de consonantes similares, en coda silábica. Como consecuencia de la aparente existencia de tal restricción en el bribri, deben buscarse indicios de ella en otras lenguas también. Por falta de datos que permitan la formulación exacta de esta restricción, la definiremos de manera amplia, sin restringirla a oclusivas:

\section{${ }^{*}$ CODA-b}

Asignar una marca de transgresión por cada consonante oral labial sonora que se encuentre en coda silábica.

Esta restricción tiene que encontrarse en el nivel más alto de la jerarquía, junto con las demás restricciones a las que se les ha asignado este rango. La Tabla 6 , en la que no se incluyen las restricciones IDENT-V́ y ALIN-I([+nas], pal), pues no son relevantes para la hipersonorización, contiene el análisis de la palabra / dabób/ [dabóm] 'diez'. 


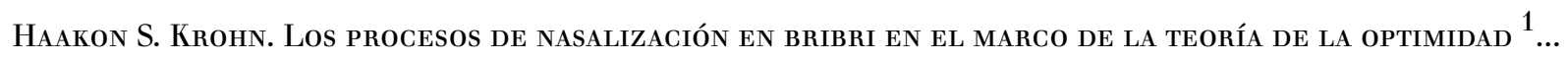

TABLA 6.

Análisis de la palabra / dabób/ 'diez'

\begin{tabular}{|c|c|c|c|c|c|c|}
\hline & /dabób/ & DEP & *CODA-b & Max & $\begin{array}{c}\text { IDENT } \\
\text { (son) }\end{array}$ & $\operatorname{DEP}([+\mathrm{nas}])$ \\
\hline a. & dabóm & & & & & $*$ \\
\hline b. & dabób & & $* !$ & & & \\
\hline c. & dabóba & $* !$ & & & & \\
\hline d. & dabó & & & $* !$ & & \\
\hline e. & dabop & & & & $* !$ & \\
\hline
\end{tabular}

Se aprecia que la restricción ${ }^{*} \mathrm{CODA}-\mathrm{b}$ es la que elimina el candidato $b$, que es fiel al aducto, frente al ganador. Por su lado, las restricciones DEP, MAX e IDENT([son]), de manera respectiva, previenen la inserción de una vocal final, la elisión de /b/ y la desonorización de /b/, tres procesos que también evitarían la presencia de /b/ en la coda. En consecuencia, $\operatorname{DEP}([+$ nas $])$ queda inactiva.

Como ya se ha señalado, en el bribri de Amubre se nasaliza cualquier consonante sonora en coda silábica cuando le precede una vocal nasal que pertenece al mismo morfema. Se podría interpretar que esto solo aplica para /d/ y / $/$, ya que el único otro fonema consonántico sonoro que puede aparecer en esta posición es /b/, cuya sonorización siempre podría ser explicada por medio de ${ }^{*} \mathrm{CODA}-\mathrm{b}$. Empero, el análisis aquí presentado mostrará que /b/ también es afectado por este proceso.

Para explicar la nasalización de consonantes sonoras en coda silábica, se podría proponer una restricción que penalizara consonantes orales sonoras detrás de una vocal nasal tautosilábica y tautomorfémica. No obstante, tal restricción no aprehendería la esencia de la asimilación, además de que contendría una combinación de mucha información específica, lo cual pondría fuertemente en duda su índole universal. Por lo tanto, en cambio, utilizaremos una restricción de alineamiento que concuerda con el carácter de propagación nasal progresiva:

ALIN-D([+nas], morf)

Asignar una marca de transgresión por cada segmento entre un segmento [+nasal] y el linde derecho de un morfema que no esté asociado con el mismo rasgo [+nasal].

En este nombre, "D” significa "derecha” y "morf” significa "morfema”, que es el dominio de esta propagación. La repercusión de esta restricción en la palabra /batsŭ́d/ [batsún] 'higuerón' se muestra en la Tabla 7. Aquí se incluyen dos candidatos con la forma superficial [batsún]; en la que lleva el subíndice 1, la nasalidad de la última consonante proviene de la vocal, mientras que en la del subíndice 2, la última consonante está asociada con un autosegmento nasal separado, como sucedería en el caso de una hipersonorización. 
TABLA 7.

Análisis de la palabra /batsû́d/ 'higuerón'

\begin{tabular}{|c|c|c|c|c|c|c|}
\hline & /batsứd/ & IDENT-V' & Max & $\begin{array}{c}\text { IDENT } \\
\text { (son) }\end{array}$ & $\begin{array}{c}\text { AlIN- } \\
\mathrm{D}([+ \text { nas }], \\
\text { morf }) \\
\end{array}$ & $\operatorname{DEP}([+$ nas $])$ \\
\hline a. & batsứn & & & & & $*$ \\
\hline b. & batsứn 2 & & & & $* !$ & $*$ \\
\hline c. & batsứd & & & & $* !$ & \\
\hline d. & batsúd & $* !$ & & & & \\
\hline e. & batsṹ & & $* !$ & & & \\
\hline f. & batsứt & & & $* !$ & $*$ & \\
\hline
\end{tabular}

Gracias a la restricción de alineamiento hacia la derecha, el candidato más fiel al aducto es eliminado antes que el ganador, que presenta una nasalización de la consonante final. Las demás restricciones incluidas en la tabla se encargan de conservar la nasalidad de la vocal tónica y prevenir la elisión y la desonorización de la última consonante. En cuanto a los candidatos $a$ y $b$, el segundo comete una transgresión a ALIN-D([+nas], morf) debido a sus dos autosegmentos [+nasal], de los que uno no llega hasta el límite derecho de la palabra.

Para apreciar cómo funciona esta restricción con respecto a consonantes sordas en la coda, las cuales no se nasalizan, se analiza la palabra /ûk/ [ûk] 'hacer' en la Tabla 8. En este caso, ${ }^{*}$ NAS/SOR y las demás restricciones se encargan de que la transgresión del ganador a la restricción de alineamiento sea irrelevante.

TABLA 8.

Análisis de la palabra /ûk/ 'hacer'

\begin{tabular}{|c|c|c|c|c|c|c|}
\hline & / $\mathrm{u} \mathrm{k} /$ & IDENT-V' & MAX & $\begin{array}{c}\text { IDENT } \\
\text { (son) }\end{array}$ & *NAS/SOR & $\begin{array}{c}\text { ALIN- } \\
\mathrm{D}([+ \text { nas }], \\
\text { morf }) \\
\end{array}$ \\
\hline a. & ûk & & & & & $*$ \\
\hline b. & Ûk & *! & & & & \\
\hline c. & $\hat{\tilde{u}}$ & & *! & & & \\
\hline $\mathrm{d}$. & $\hat{\tilde{u}}$ & & & $* !$ & & \\
\hline e. & uิ๊o & & & & $* !$ & \\
\hline
\end{tabular}

En las tablas anteriores, los fenómenos de armonía nasal y la nasalización de consonantes en coda silábica han sido tratados por separado. Sin embargo, ciertas palabras presentan ambos fenómenos simultáneamente, una de las cuales es /kadếb/ [kã̃̂̃̂́m] 'pez barbudo'. En la Tabla 9 se analiza este vocablo. 


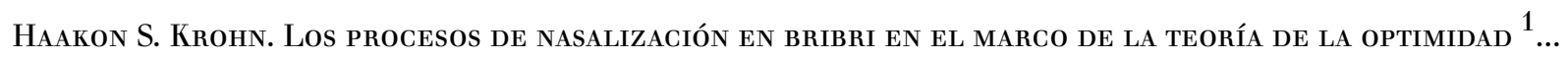

Se incluyen solamente las restricciones ${ }^{*}$ CODA-b, ALIN-I([nasal], pal) y ALIN-D ([+ nas], morf), con el fin de destacar la interacción entre las restricciones que son responsables de los procesos en cuestión. Está claro que *CODA-b tiene que dominar a ALIN-I([nasal], pal), pues la primera es la única de las dos que nunca es transgredida por candidatos ganadores, como se ha mencionado anteriormente. Asimismo, ALIN$\mathrm{D}([+$ nas $]$, morf $)$ se coloca al mismo nivel que ALIN-I([nasal], pal), pues no hay manera de determinar la jerarquización respectiva entre estas dos: con cualquier orden, el ganador es el mismo. De nuevo, se incluyen candidatos con subíndices; 1 significa que presenta un solo autosegmento nasal y 2 indica que presenta dos (uno que tiene su origen en la última vocal y uno asociado con la última consonante).

TABLA 9.

Análisis de la palabra /kadếb/ 'pez barbudo'

\begin{tabular}{|c|c|c|c|c|}
\hline & /kadẽ́b/ & ${ }^{*}$ CODA-b & $\begin{array}{c}\text { ALIN- } \\
\text { I([nasal] } \\
\text { pal })\end{array}$ & $\begin{array}{c}\text { ALIN- } \\
\mathrm{D}([+ \text { nas }], \\
\text { morf }) \\
\end{array}$ \\
\hline a. & kãrếm1 & & $*$ & \\
\hline b. & kãrếm2 & & $* * ! * * *$ & $* !$ \\
\hline c. & kadếb & $* !$ & $* * *$ & $*$ \\
\hline d. & kãr̃ếb & $* !$ & $*$ & $*$ \\
\hline e. & kadếm1 & & $* * ! *$ & \\
\hline f. & kadếm2 & & $* *$ !***** & $* !$ \\
\hline
\end{tabular}

La alta posición de ${ }^{*}$ CODA-b en la jerarquía asegura que se eliminen los candidatos con [b] final, y ALIN$\mathrm{I}([$ nasal $]$, pal) favorece a los candidatos con armonía nasal que llega hasta la consonante bloqueadora $/ \mathrm{k} / \mathrm{El}$ hecho de que el candidato $a$, en el que $[\tilde{e}]$ y $[\mathrm{m}]$ comparten un mismo autosegmento nasal, le gane al candidato $b$ significa que la nasalización de / b/ en coda silábica tiene que clasificarse como un resultado de propagación nasal hacia la derecha, y no como hipersonorización, cuando la vocal precedente es nasal.

El último fenómeno por analizar es la nasalización del primer segmento en geminaciones presentes en formas históricamente reduplicadas en el dialecto de Amubre, la cual es otra instancia de hipersonorización. Los fonemas afectados son $/ \mathrm{b} / \mathrm{y} / \mathrm{d} /(/ \mathrm{r} /$ nunca se gemina, ya que nunca se halla en ataque silábico), pero ALIN-D ([+nas], morf $)$ es irrelevante aquí, pues la nasalización no depende de una vocal nasal. En cambio, lo que produce la nasalización en estas formas es claramente una prohibición de consonantes sonoras geminadas, la cual es respetada por medio de una nasalización del primero de los dos segmentos. Por lo tanto, parece estar involucrada la siguiente restricción (véase Podesva, 2002):

\section{${ }^{*}$ GemSon}

Asignar una marca de transgresión por cada consonante sonora geminada. 
Esta restricción tiene que encontrarse en el nivel más alto de la jerarquía, puesto que siempre es respetada. Ahora bien, cabe considerar por qué siempre es la primera, y no la segunda, consonante la que se nasaliza. Existen varias posibles explicaciones que involucran restricciones adicionales; sin embargo, la jerarquización relativamente alta de ALIN-I ([+nas], pal), que originalmente propusimos para un fenómeno aparentemente no relacionado, resuelve este problema de manera elegante, como se observa en la Tabla 10, en la que se analiza la palabra /díddí/ [díndí] 'afilado'.

En lo que concierne a la restricción ALIN-D $([+$ nas $]$, morf $)$, esta es muy difícil de incluir en el presente análisis, debido a que el estatus morfemático de la segunda /d/ es difuso; aun si se asume que las dos raíces siguen siendo dos morfemas distintos a nivel sincrónico de la lengua, no está claro si dicho fonema forma parte del segundo morfema o si es un morfema por sí solo. Dado que el dominio de la propagación hacia la derecha es el morfema, la cantidad de violaciones a ALIN-D ([+nas], morf $)$ depende fuertemente del análisis morfológico de esta palabra. Lo que sí es seguro es que si la segunda /d/ pertenece al mismo morfema que la última instancia de /dí/, ALIN-D([+nas], morf) no puede dominar a ALIN-I([+nas], pal), ya que se eliminaría a [díndí] antes que a [díní]. En cambio, en caso de que este fonema se encuentre en posición final de morfema, el orden respectivo de las dos restricciones de alineamiento es irrelevante para el análisis de esta palabra, puesto que [díní] violaría ambas restricciones una mayor cantidad de veces que [díndí]. En resumen, dado que el número de marcas de transgresión para ALIN-D ([+nas], morf) es inseguro y la posición de esta restricción con respecto a $\mathrm{ALIN}-\mathrm{I}([+$ nas $]$, pal) en cualquier caso sería imposible de determinar con base en el análisis de /díddí/, no se incluye en la Tabla 10.

TABLA 10.

Análisis de la palabra / díddí/ 'afilado'

\begin{tabular}{|c|c|c|c|c|c|c|}
\hline & /díddí/ & DEP & Max & *GEMSON & $\begin{array}{c}\text { ALIN- } \\
\mathrm{I}([+ \text { nas }], \\
\text { pal }) \\
\end{array}$ & $\operatorname{DEP}([+$ nas $])$ \\
\hline a. & díndí & & & & $* *$ & * \\
\hline b. & díddí & & & $* !$ & & \\
\hline c. & dírní & & & & $* * * !$ & $*$ \\
\hline $\mathrm{d}$. & dínní & & & $* !$ & $* *$ & $* *$ \\
\hline e. & dídadí & $* !$ & & & & \\
\hline f. & dídí & & $* !$ & & & \\
\hline g. & níndí & & & & $* *$ & $* * !$ \\
\hline
\end{tabular}

Aquí se aprecia, en primer lugar, que DEP y MAX previenen la elisión de una de las dos consonantes y la inserción de una vocal entre ambos, como ocurre en los candidatos e y $f$, respectivamente. En segundo lugar, la nasalización de ambas, como en $d^{13}$, tampoco funciona, ya que sigue habiendo una consonante sonora geminada. Por lo tanto, los tres candidatos restantes son $a, c$ y $g$. En $a$ y $g$, la primera consonante se nasaliza, y en $c$, la segunda se nasaliza (y la primera / $\mathrm{d} /$ se pronuncia $[\mathrm{r}$ ], debido a una restricción relativa a la coda silábica que no es tratada en este artículo). La nasalización de una consonante genera un autosegmento [+nasal], por lo que entra en juego ALIN-I([+nas], pal), cuya definición no implica que el segmento desencadenador tenga que ser una vocal. Debido a que esta restricción incita una propagación hacia la izquierda, los candidatos 


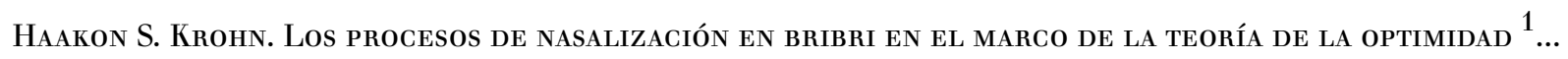

que nasalizan a la primera de las dos consonantes cometen una transgresión menos que el otro. Al final, el candidato $g$ es eliminado como resultado de las dos nasalizaciones de fonemas orales.

En síntesis, parece que la restricción responsable de la armonía nasal regresiva también es la que define cuál de las dos consonantes geminadas se nasalice, lo cual significaría que existiría un vínculo inesperado entre la armonía nasal y la nasalizacióm en palabras reduplicadas. Desde luego, es perfectamente posible que alguna otra restricción sea la responsable de este detalle, pero los datos no permiten identificarla de manera precisa.

Por último, en cuanto a las secuencias /bb/ en palabras reduplicadas, tanto *GEMSON como *CODA-b, que se encuentran en el mismo nivel de la jerarquía, propulsan la nasalización. Como se observa en la Tabla 11, en la que se analiza /bâbbá/ [bâmbá] 'caliente', ALIN-I([+nas], pal) no es necesaria para definir cuál de las dos consonantes se nasaliza en este caso, pues ${ }^{*}$ CODA-b siempre favorece la nasalización de la primera.

TABLA 11.

Análisis de la palabra /bâbbá/ 'caliente'

\begin{tabular}{|c|c|c|c|c|c|c|c|}
\hline & /bâbbá/ & DEP & MAX & *GEMSON & $*$ CODA-b & $\begin{array}{c}\text { ALIN- } \\
\mathrm{I}([+ \text { nas }], \\
\text { pal })\end{array}$ & $\operatorname{DEP}([+$ nas $])$ \\
\hline a. & bâmbá & & & & & $* *$ & * \\
\hline b. & bâbbá & & & *! & $* !$ & & \\
\hline c. & bâbmá & & & & $* !$ & $* * *$ & $*$ \\
\hline d. & bâmmá & & & $* !$ & & $* *$ & $* *$ \\
\hline e. & bâbabá & $* !$ & & & & & \\
\hline f. & bâbá & & $* !$ & & & & \\
\hline
\end{tabular}

\section{Discusión}

Por medio de los análisis de distintos tipos de nasalización en bribri, hemos determinado que las siguientes restricciones se encuentran en el primer nivel de la jerarquía, puesto que nunca son transgredidas: IDENT-V́, DEP, MAX, IDENT(son), *NAS/SOR, *GEMSON y *CODA-b. Por debajo de estas, se encuentran dos restricciones de alineamiento, cuyo orden respectivo no es posible de definir con base en los datos presentados: ALIN-I ([+nas], pal) y ALIN-D([+nas], morf). En el último nivel de las restricciones analizadas se encuentra $\mathrm{DEP}([+$ nas $])$. Por lo tanto, la jerarquización identificada en esta investigación es la siguiente:

IDENT-V́, DEP, MAX, IDENT(son), ${ }^{*} \mathrm{NAS} / \mathrm{SOR},{ }^{*} \mathrm{GEMSON},{ }^{*} \mathrm{CODA}-\mathrm{b}>>$ ALIN-I([+nas], pal), ALIN-D([+nas], morf $)>>D E P([+$ nas $])$

En cuanto a la propagación nasal en el bribri, esta se inserta en un continuo de distintos patrones observadas en las lenguas del mundo (Walker, 2011), con diferentes valores para todos los parámetros presentados en el apartado 2.2, así como para el dominio de propagación. Por ejemplo, la direccionalidad asimétrica de la propagación en bribri, en la que cada dirección sigue patrones diferentes, ya ha sido identificada en otras lenguas (Walker, 2011, p. 21). Lo más llamativo es, probablemente, el hecho de que el conjunto de segmentos bloqueadores no concuerde con la jerarquía de sonoridad, lo cual parece ser lo más común a nivel interlingüístico (véase Walker, 1998, p. 36; 2011, p. 9), sino que los bloqueadores sean definidos por restricciones independientes de dicha jerarquía: IDENT-V y *NAS/SOR. Por su lado, la nasalización por 
hipersonorización es una de varias posibles consecuencias de la prohibición de determinadas consonantes en posiciones periféricas.

\section{Conclusiones}

En este artículo se ha realizado un análisis teórico-óptimo de datos ya conocidos del bribri. Esta aproximación permite visualizar los procesos de nasalización desde una óptica distinta y vincularlos con fenómenos en otras lenguas por medio de las restricciones que se manifiestan: la jerarquización de las restricciones involucradas en los procesos de nasalización en bribri sería justamente la que determina la ubicación del bribri en el espacio tipológico-fonológico de las lenguas del mundo. Aunque la mayoría de las restricciones activas en los procesos de nasalización en bribri han sido identificadas anteriormente en otras lenguas, algunas son más específicas, por lo que la identificación de estas en futuras investigaciones respaldaría nuestro análisis. En definitiva, más fenómenos de otras lenguas chibchenses deben analizarse dentro del marco de la teoría de la optimidad, ya que esta familia lingüística indudablemente tiene datos muy valiosos que ofrecerle a esta teoría de restricciones fonológicas universales.

\section{BibLIOGRAFía}

Chevrier, N. (2017). Analyse de la phonologie du bribri (chibcha) dans une perspective typologique. Nasalité et géminée module. (Tesis doctoral). Université Lumière Lyon 2.

Chomsky, N. y Halle, M. (1968). The Sound Patterns of English. Nueva York: Harper \& Row.

Constenla Umaña, A. (1981). Comparative Chibchan phonology. (Tesis doctoral). University of Pennsylvania.

Constenla Umaña, A. (1982). La nasalización en relación con el tono y la intensidad en bribri. Revista de Filología y Lingüistica de la Universidad de Costa Rica, 1-2, 109-120.

Constenla Umaña, A. (1985). Prosodic nasality in Bribri (Chibchan) and Universals of Nasality. International Journal of American Linguistics, 51, 374-376.

Constenla Umaña, A. (2008). Estado actual de la subclasificación de las lenguas chibchenses y de la reconstrucción fonológica y gramatical del protochibchense. Estudios de Lingüistica Chibcha, 27, 117-135.

Constenla Umaña, A. (2012). Chibchan languages. En L. Campbell y V. Grondona (Eds.), The Indigenous Languages of South America (pp. 391-439). Berlín/Boston: De Gruyter Mouton.

Constenla Umaña, A., Elizondo Figueroa, F. y Pereira Mora, F. (1998). Curso básico de bribri. San José: Editorial de la Universidad de Costa Rica.

Goldsmith, J. (1976a). Autosegmental Phonology. (Tesis doctoral). Massachusetts Institute of Technology.

Goldsmith, J. (1976b). An overview of autosegmental phonology. Linguistic Analysis, 2, $23-68$.

Gussenhoven, C. (2004). The Phonology of Tone and Intonation. Cambridge: Cambridge University Press.

Jara Murillo, C. V. (2004). Observaciones para el estudio dialectológico de la lengua bribri. Estudios de Lingüistica Chibcha, 23, 89-120.

Jara Murillo, C. V. (2018). Gramática de la lengua bribri. San José: E-Digital.

Kristoffersen, G. (2000). The Phonology of Norwegian. Oxford: Oxford University Press.

Maddieson, I. (2013). Absence of common consonants. En M. S. Dryer y M. Haspelmath (Eds.), The World Atlas of Language Structures Online. Leipzig: Max Planck Institute for Evolutionary Anthropology. Recuperado de htt ps://wals.info/chapter/18

McCarthy, J. J. (2004). Headed spans and autosegmental spreading. (Manuscrito no publicado). University of Massachusetts Amherst.

McCarthy, J. J. (2008). Doing Optimality Theory. Applying Theory to Data. Malden/Oxford/Carlton: Blackwell Publishing. 


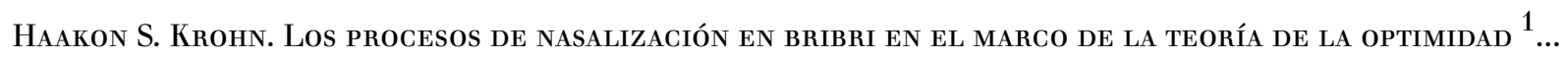

McCarthy, J. J. (2011). Autosegmental spreading in Optimality Theory. En J. A. Goldsmith, E. Hume y L. Wetzels (Eds.), Tones and Features. Phonetic and Phonological Perspectives (pp. 195-222). Berlín/Boston: De Gruyter Mouton.

McCarthy, J. J. (2016). The theory and practice of Harmonic Serialism. En J. J. McCarthy y J. Pater (Eds.), Harmonic Grammar and Harmonic Serialism (pp. 47-87). Sheffield: Equinox Publishing.

Ohala, J. D. (2011). Accomodation to the aerodynamic voicing constraint and its phonological relevance. Proceedings of the 17th International Congress of Phonetic Sciences, 64-67.

Ohala, J. D. y Solé, M.-J. (2008). Turbulence \& Phonology. UC Berkeley PhonLab Annual Report, 4(4), $297-355$.

Pache, M. J. (2018). Contributions to Chibchan historical linguistics. (Tesis doctoral). Universiteit Leiden.

Podesva, R. J. (2002). Segmental constraints on geminates and their implications for typology. Handout de la $76 t h$ LSA Annual Meeting. San Francisco, California.

Prince, A. y Smolensky, P. (1993/2004). Optimality Theory: Constraint Interaction in Generative Grammar. Malden/ Oxford: Blackwell.

Schlabach, R. (1974). Los fonemas del bribri del Valle de Talamanca. América Indígena, 34(2), 355-362.

Tohsaku, Y. H. (1987). Bribri nasal harmony from the vantage point of the Universal Theory of Harmony. Working Papers of the Linguistics Circle of the University of Victoria, 6(1), 1-10.

Walker, R. L. (1998). Nasalization, neutral segments, and opacity effects. (Tesis doctoral). University of California, Santa Cruz.

Walker, R. L. (2011). Nasal harmony. En M. Oostendorp, C. Ewen, E. Hume y K. Rice (Eds.), The Blackwell Companion to Phonology (pp. 1873-1900). Malden: Wiley-Blackwell.

Wilson, C. (2003). Unbounded spreading in OT (or, Unbounded spreading is local spreading iterated unboundedly). Handout de SWOT. Tucson, Arizona.

Wilson, C. (2004). Analyzing unbounded spreading with constraints: marks, targets, and derivations. (Manuscrito no publicado). University of California, Los Angeles.

Wilson, C. (2006). Unbounded spreading is myopic. Handout de Phonology Fest 2006. Bloomington, Indiana.

Wilson, J. L. (1970). Oclusivas sonoras nasalizadas en bribri. Revista de la Universidad de Costa Rica, 29, $159-163$.

Wilson, J. L. (1974). Análisis fonológico del bribri. América Indígena, 34(2), 419-422.

Wilson, J. L. (1982). El alfabeto bribri. En E. Margery Peña (Ed.), Diccionario fraseológico bribri-español español-bribri (pp. xi-xx). San José: Editorial de la Universidad de Costa Rica.

\section{Notas}

1 Este artículo forma parte del proyecto de investigación 745-C0-200 “Diccionario digital bilingüe bribri (fase 2)”, inscrito en el Instituto de Investigaciones Lingüísticas de la Universidad de Costa Rica.

2 Específicamente, forma parte de la subrama viceítica de la rama ístmica de dicha familia lingüística (Constenla, 2008, 2012).

3 En varias descripciones del bribri, los fonemas aquí presentados como /e/y /o/ se representan con los símbolos / ع/ y / /, respectivamente. Sin embargo, ya que no existe contraste fonológico entre vocales semicerradas y semiabiertas en esta lengua, así como por el hecho de que no se han publicado estudios acústicos que justifiquen esta caracterización fonética, optamos por emplear los símbolos más básicos /e/ y /o/.

4 De acuerdo con nuestras propias observaciones, esta consonante no es alveolar, sino retrofleja, tal como afirma Chevrier (2017).

5 Comúnmente, el término acento tonal se utiliza con referencia a la entonación. Este no es el caso aquí, por lo que no debe confundirse con lo que en inglés se conoce como "pitch accent", ya que, en el bribri, el acento tonal es fonológico. Por lo tanto, el término se emplea en este artículo en el mismo sentido en el que Kristoffersen (2000, pp. 233-234) lo utiliza para los acentos tonales fonológicamente contrastivos del noruego y el sueco.

6 Jara (2004, p. 100), a su vez, reporta un trío mínimo de palabras distinguidas únicamente por el tono en el bribri de Coroma. Sin embargo, se trata de un solo caso, por lo que, como ella misma señala, esto tendrá que estudiarse con mayor detalle. 
Revista de Filología y Lingüística de la Universidad de Costa Rica, 2021, vol. 47, núm. 2, e46626, ...

7 En este primer conjunto de ejemplos, no se señalan los lindes morfemáticos, puesto que son irrelevantes para el fenómeno ilustrado.

8 Por ejemplo, para la palabra /uîb/ 'hombre', Chevrier (2017, pp. 272-275) registra las pronunciaciones [wîm], [wîb] y [wîmb] en posición intermedia de enunciado en el bribri de Amubre, y solo [wîm] en el de Coroma.

9 La tabla de Chevrier (2017, p. 169) incluye también los fonemas /w/y /j/, los cuales nosotros no consideramos fonemas, sino alófonos de /u/ e /i/.

10 Seguimos la recomendación de McCarthy (2008) de comenzar cada definición con "Asignar una marca de transgresión por cada...”, con el fin de que sea lo más precisa posible.

11 Específicamente, una restricción que penalice vocales pretónicas.

12 Interesantemente, Ohala (2011, p. 66) cuestiona de manera explícita la aplicación de la teoría de la optimidad para explicar los fenómenos de hipersonorización, principalmente por la dificultad de imaginarse la AVC representada por una restricción general en dicha teoría. Sin embargo, la crítica está formulada más que nada como una aversión general contra la teoría de la optimidad, y es difícil ver por qué los distintos tipos de hipersonorización serían menos adecuados para ser descritos en el marco de esta teoría que otros fenómenos fonológicos.

13 Asumimos aquí que, por tratarse de una consonante geminada, ambos segmentos comparten el mismo autosegmento [+nasal]. Sin embargo, aunque el caso contrario provocaría una mayor cantidad de marcas de transgresión para la restricción ALIN-I([+nas], pal), esto nunca sería determinante para elección del candidato ganador. 